\title{
Early Intervention IN PSYCHIATRY
}

\section{Spatial working memory, not IQ or executive function discriminates early psychosis and clinically vulnerable creative individuals}

\begin{tabular}{|r|l|}
\hline Journal: & Early Intervention in Psychiatry \\
\hline Manuscript ID & EIP-2019-102.R1 \\
\hline Danuscript Type: & Original Article \\
\hline Complete List of Authors: & $\begin{array}{l}\text { Crabtree, Julie; University of Technology Sydney, Graduate School of } \\
\text { Health: discipline of clinical psychology } \\
\text { Newton-John, Toby; University of Technology Sydney, Graduate School } \\
\text { of Health: discipline of clinical psychology } \\
\text { Hudson, Jennie; Macquarie University, Director, Centre for Emotional } \\
\text { Health, Department of Psychology } \\
\text { Brockman, Robert; Australian Catholic University Faculty of Health } \\
\text { Sciences, Institute for positive psychology and education }\end{array}$ \\
\hline Keywords: & Creativity, Cognition, Early psychosis, Spatial working memory \\
\hline &
\end{tabular}

\section{SCHOLARONE




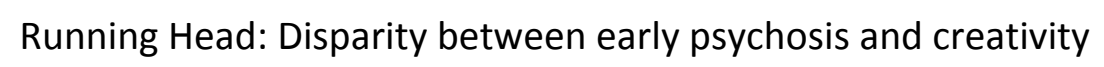

Spatial working memory, not IQ or executive function, discriminates early psychosis and clinically vulnerable creative individuals 17 (1) (1) (1) (1) Julie Crabtree ${ }^{\mathrm{a}}$, Jennifer L. Hudson ${ }^{\mathrm{b}}$, Robert Brockman ${ }^{\mathrm{c}}$, Toby Newton-John a

\section{November 2019}

Word count: 4670

Abstract: 247

Text:

Figures:

Tables:

*Corresponding author:

Julie Crabtree

juliecrabtree@mac.com

a Graduate School of Health

University of Technology Sydney.

b Centre for Emotional Health, Department of Psychology

Macquarie University, Sydney.

41 Australian Catholic University, Sydney.

42 Abstract

42 Abstract 
1 Aim: While associations between creativity and psychopathology have been well researched, the specific cognitive processes that distinguish highly creative from those with psychopathology warrant further investigation. This study will examine whether IQ, executive function, cognitive inhibition or spatial working memory differentiate individuals with early psychosis, clinically vulnerable creative individuals, creative controls and non-creative controls.

Methods: The study sample consisted of 110 participants: early psychosis $(n=21)$; clinically vulnerable creative controls $(n=25)$; creative controls $(n=30)$ and noncreative control $(n=34)$. The Diagnostic Interview for Psychosis assessed early psychosis participants and the Mini Neuropsychiatric Interview was used to screen for psychopathology in the remaining groups. Several cognitive tests were administered: $I Q$, neurocognitive measures of executive function and spatial working memory. Creativity was assessed using the Torrance Test of Creativity and Creative Achievement Questionnaire. A measure of vividness of mental imagery was also given.

Results: Across all cognitive tests, spatial working memory differentiated the early psychosis group from both creative and non-creative control groups. Spatial working memory predicted group membership but vivid imagery was a better predictor of creative achievement. The early psychosis, clinically vulnerable creative and creative groups all recorded significantly higher results on creative achievement and creative cognition compared to non-creative controls.

Conclusions: Our results provide further support for spatial working memory as an early neuro-cognitive marker for early psychosis. Spatial working memory, rather than IQ or executive function, may also be an early protective factor for clinically vulnerable young creative individuals.

Keywords: Creativity, Cognition, Early psychosis, Spatial Working Memory 
The association between creativity and psychopathology has been the subject of speculation and conjecture since antiquity when Aristotle first commented on melancholy among those who were prominent in society at the time (Becker, 2014). More recent studies provide evidence of a strong link between creativity and mood disorder and thought disorder similar to psychotic thinking (Rybakowski, Klonowska, Parrzala, \& Jaracz, 2008). This association has been corroborated in recent genetic and epidemiological studies, which suggest shared genetic roots between creative professionals and those with a diagnosed psychosis (Kyaga et al., 2011; Parnas, Sandsten, Vestergaard, \& Nordgaard, 2019). Notably, epidemiological evidence suggests that this association is particularly found between artistic creativity and psychosis. A recent large population based case-controlled study (MacCabe et al., 2018) found that students who studied artistic subjects (such as art and performance) at university had significantly increased odds of developing schizophrenia and bipolar disorder. Moreover the author's conclude that the association between creativity and psychopathology represents risk factors similar to other identified risk factors for psychotic illness. Similarly, a genetic study from Iceland (Power et al., 2015) reported that both bipolar and schizophrenia polygenic risk scores were associated with professional creative artists, and that this association was not found in five other professions. Although research in this area has focused on the similarities between creativity and psychopathology (Claridge \& Blakey, 2009), the question of what enables a young artistic, creative student to follow a career of creative expression rather than a trajectory of pathology invites a closer examination of possible cognitive distinguishing factors that may protect young creative artists from psychopathology (Carson, 2011). Although previous research has examined some aspects of cognition, visuo-cognitive factors have not been studied. This investigation into what cognitive (including visuo-cognitive) similarities and differences exist between the highly creative individual and those with early psychosis is of increased importance as it may lead to innovative treatments and early remediation of those presenting as at risk for psychosis and young clinically vulnerable creative individuals.

\section{Cognitive and schizotypal similarities between artistic creative and psychosis} populations

A number of studies have found personality and cognitive similarities between the creative and those with psychopathology, with similarities in schizotypy in particular

40 extensively examined. Schizotypy refers to aspects of personality and perceptual 41 beliefs that indicate a person's proneness to schizophrenia and psychosis (Siddi, 42 Petretto, \& Preti, 2017). It is usually defined as three factors: positive, negative and 
1 disorganised (Debbané et al., 2015) with creativity strongly associated with positive

2 schizotypy, namely impulsive non-conformity and unusual experiences (Ando,

3 Claridge, \& Clark, 2014; Nelson \& Rawlings, 2010; Nettle, 2006). With respect to

4 cognition, similarities in divergent thinking (Claridge \& Blakey, 2009; Srivastava et al., 5 2010) and cognitive inhibition (Beech, Powell, McWilliam, \& Claridge, 1989; Carson,

6 Peterson, \& Higgins, 2003) have been reported in both highly creative and psychosis 7 populations (Richards, 2001; Sass \& Schuldberg, 2001). Divergent thinking is rapid, 8 fluid thinking in which the person is able to make unique associations (Guilford, 9 1967; Torrance, 1993) and underlies both schizotypal and manic thought (Claridge \& 10 Blakey, 2009; Srivastava et al., 2010). Decreased cognitive inhibition, or the reduced 11 ability to filter out irrelevant information and bring more stimuli into conscious 12 awareness, is thought to be a process underlying divergent thinking (Eysenck, 1994) 13 and has been linked with both schizotypy and creativity (Brod, 1997; Ottemiller, 14 Elliott, \& Tania, 2014).

Proposed cognitive differences between artistic creative and psychosis populations:

\section{$171 Q$, executive function}

General intellectual functioning (IQ) has been mooted as a possible cognitive protective factor for highly creative individuals (Carson, 2011; Rybakowski et al., 2008) where it is thought that those with high IQ are better able to process and manipulate additional stimuli arising from reduced cognitive inhibition. However, few studies support this assertion (Kyaga et al., 2011; Miller \& Tal, 2007; Soeiro-deSouza, Dias, Bio, Post, \& Moreno). If not high IQ, then executive function may differentiate the two populations. Executive functions are those subserved by the frontal lobes of the brain and refer to cognitive skills such as planning, organising, and shifting mental set (often termed cognitive flexibility) (Baddeley, 1996). However, while executive function deficits are found in both bipolar and schizophrenia populations (Bora, Yucel, \& Pantelis, 2009), age (Fucetola et al., 2000), onset and duration of illness are key factors in assessing executive function. Rather than declining executive function, a meta-analysis of cognitive deficits in ultra-high risk and early psychosis populations found stable and improved executive function across both "at risk" and early psychosis populations (Bora \& Murray, 2013). This research suggests that executive function deficits are not as evident in "at risk" or early stage psychosis, which is the population of most concern in discriminating possible risk factors in creative arts students.

Executive function in the context of creativity and psychosis has been investigated in one previous study. Soeiro-de-Souza and colleagues (2011) tested a group of 18-35

40 year old, un-medicated participants experiencing manic, depressed and mixed

41 (mania and depression) episodes. In this study, creativity was higher in manic and 42 mixed states, compared to those experiencing a depressive episode and importantly, 
1 higher creativity was associated with greater executive function in manic state only.

2 IQ did not influence creativity across all three, mood states. Based on this study,

3 where the manic group had elevated creativity and executive function, early indicators are that executive function may not differentiate the creative from those with psychopathology. However, a comparison of executive function in early psychosis and creative populations would provide more clarity of the role of executive function as a protective factor for the creative population. Notwithstanding there are additional visuo-cognitive factors that warrant attention.

Visuo-cognitive factors: Does spatial working memory differentiate between artistic creativity and psychosis?

One important area of cognitive functioning that has received relatively less research attention in understanding the creativity/psychopathology nexus is visuospatial working memory. Visuospatial working memory refers to the ability to keep spatial information active for a period of time, involving spatial orientation and the recording of information about one's environment. Although not investigating creativity specifically, a group of researchers (Goghari et al., 2014), examined the role of spatial working deficits and IQ in those at ultra-high risk (UHR) for psychosis and first episode psychosis patients (FEP), compared to controls (HC). All three groups were compared on WASI Verbal and Performance IQ (Canivez, Konold, Collins, \& Wilson, 2009), and spatial working memory. The spatial working memory (SWM) task was taken from Cambridge Neuropsychological Test Automated Battery (CANTAB) (Sahakian \& Owen, 1992) and requires retention and manipulation of visuospatial material. The SWM task is able to differentiate two of the components of working memory, namely the short-term maintenance of material (in the visuospatial sketchpad) and the use of task strategy. This study found that, controlling for IQ the short-term maintenance of material in the visuospatial sketchpad differentiated the UHR from the HC groups. Furthermore, it was impaired SWM, rather than IQ, that significantly predicted global functioning in both the UHR and FEP groups. The assertion that deficits in spatial working memory may be an early risk factor for psychosis and a more reliable neurocognitive marker for psychosis, compared to other cognitive factors, (Carrión et al., 2018), has been supported in other studies (Badcock, Michiel, \& Rock, 2005; Brewer et al., 2006; Pirkola et al., 2005). In addition to this, a number of recent reviews have proposed that aberrations in visual functioning, such as deficits in spatial working memory are associated with early psychosis (Landgraf \& Osterheider, 2013; Uhlhaas \& Mishara, 2007), which may in turn drive delusion formation (Grillo, 2018). Further research into these visuo-cognitive impairments is needed. Whilst the role of spatial working memory as a possible vulnerability marker for psychosis is an emerging area of research, its relationship to creativity has not been examined. 
1 Similarities in vividness of mental imagery in artistic creative and psychosis

\section{populations}

Associated with spatial memory and common to both creative and psychosis populations is enhanced vividness of mental imagery. Vivid mental imagery is defined as the degree of perceptual detail experienced in visualising scenes and objects (Oertel et al., 2009). It has been associated with hallucinations in schizophrenia and has been proposed as a trait marker for schizophrenia (Sack, van de Ven, Etschenberg, Schztz, \& Linden, 2005). Moreover it is one of the indicators of creativity (Michalica \& Hunt, 2013; Thomson \& Jaque, 2018; Wang et al., 2017). It may be that there is also a relationship between those with strong mental imagery and spatial working memory such that those with heightened mental imagery may utilise it to aid performance in visuospatial working memory tasks (Keogh \& Pearson, 2011). Aspects of this association have been examined in recent studies using small clinical populations and healthy controls (Benson \& Park, 2013; Matthews, Collins, Thakkar, \& Park, 2014). Benson and Park (2013) investigated visuospatial ability and mental imagery in a population of eighteen patients with schizophrenia (Scz) matched to eighteen controls. The clinical population demonstrated enhanced mental imagery but impaired spatial working memory, indicating that the clinical population were unable to recruit their superior mental imagery in spatial working memory tasks. No studies have explored this question in a creative and early psychosis population and this will be investigated in this study.

\section{Aims}

The aims of this study are to investigate whether spatial working memory rather than IQ and measures of executive function differentiate creative and non-creative controls, from early psychosis populations. We hypothesise that IQ, cognitive inhibition and executive function will not differentiate early psychosis and control groups however early psychosis participants will record greater SWM deficits than both control groups. Furthermore the early psychosis cohort will retain similarly enhanced vividness of mental imagery and creative cognition but not creative achievement compared to creative controls.

\section{Methods}

Recruitment and study procedures were approved by the Human Research Ethics Committees of the University of New South Wales (HREC UNSW Protocol No. 11279) and ratified by University of Technology (HREC UTS 16-0532). All participants provided written consent prior to participation. As the study sought to focus on first episode psychosis, only participants aged between 18-35 were recruited. All participants were given an AUD\$40 monetary reimbursement for travel costs. 


\subsection{Participants}

3

\section{Early psychosis participants (EP) $N=21$}

Early psychosis participants were recruited from a previous study (Rowland et al., 2012), and had given consent to be approached for further studies. All were recruited from either an inpatient or outpatient hospital clinic and had undergone a psychiatric assessment using a comprehensive Diagnostic Interview for Psychosis (DIP)(Castle DJ et al., 2006), administered by an experienced clinician. All early psychosis participants met criteria for psychotic disorder according to ICD -10 criteria and had recorded a first episode psychosis or hospitalisation. All were under regular psychiatric supervision. Active psychotic symptoms were an exclusion criterion for the study (see table 1 for PANSS scores), however no participants were excluded due to the presence of acute psychotic symptoms.

\section{Creative control participants $(C C) \mathrm{N}=30$}

Creative control participants were recruited from several Creative Art Colleges around Sydney, Australia. The recruitment sites included tertiary creative training institutions; music colleges (contemporary, classical music, composition, vocals), visual arts colleges, as well as advertising on relevant creative websites (such as livingwithacreativemind.com). Creative controls were screened for past or present psychotic symptoms using a MINI Plus 5.0 (structured psychiatric questionnaire) (Lecrubier et al., 1997). None were excluded for past or present syndromal psychiatric disorders.

\section{Clinically vulnerable creative control (CVCC) $N=25$}

In administering the MINI Plus 5.0 structured psychiatric interview, it was observed that twenty-five of the creative participants reported past symptoms of hypomania or psychosis symptoms that failed to reach criteria for diagnosis. Due to the high number of creative participants who reported these symptoms, it was decided to undertake further analysis and consider this a possible clinically vulnerable creative group. Assessment of clinically vulnerable and at risk mental states for psychosis is commonly undertaken in help seeking patients who attend outpatient or inpatient services where symptoms can be assessed over time (Brewer et al., 2006). In contrast this clinically vulnerable creative group was not treatment seeking and recruited as a control group from several creative arts institutions and therefore could not be considered to satisfy the normal criteria for "at risk" mental states (Yung et al., 2005).

However as schizotypal personality is one of the CAARMS (Comprehensive assessment of "at risk" mental states) vulnerability factors for determining "at risk" 
1 for psychosis (Debbané et al., 2015; Yung et al., 2005), we examined schizotypy

2 scores taken from the O-Life and Paranoia Suspiciousness scale. Only the clinically

3 vulnerable creative group (CVCC) had a schizotypal profile that was more aligned to

4 the early psychosis group (EP), when compared to the creative and non-creative

5 groups (see appendix 1). Both CVCC and EP recorded significantly higher scores on

6 positive negative and disorganised schizotypy compared to creative and non-creative

7 participants. These findings lend support for the conjecture that this clinically

8 vulnerable creative group may be more closely related to the early psychosis

9 population. It was therefore decided to investigate this group as a separate more

10 vulnerable creative subgroup. Moreover it was hypothesised that this group may be

11 more cognitively aligned to the early psychosis population.

Non-creative control participants (NCC) $N=34$

14 Healthy, non-creative controls were recruited via a university website. The online

15 information asked for psychologically healthy volunteers to participate in research

16 into creative cognition, and thirty-three individuals initially responded. Nine

17 participants were excluded because they met either of the following three exclusion

18 criteria: i) no personal or family history of mental illness; ii) an inability to

19 communicate proficiently in spoken and written English and iii) receipt of more than

20 rudimentary training (defined as three years of less) in any field of creative arts.

21 Using the MINI Plus 5.0 (Lecrubier et al., 1997) the normal non-creative controls

22 were also screened for any current mental illness and one participant was excluded

23 (presence of an eating disorder). These participants were reimbursed \$AUD 40 or

24 their time.

\subsection{Measures}

All participants completed a series of tests including cognitive measures, self-report questionnaires, mood assessment and intellectual ability testing.

\subsubsection{Intelligence Quotient -Weschler Abbreviated Scale of Intelligence (WASI)}

32 (Wechsler \& Zhou, 2011)

33 In order to test for IQ, the two-scale version of the WASI was administered to all 34 participants.

\subsubsection{Executive function (cognitive flexibility)- Intra-extra Dimensional Shift (IED)}

37 (Sahakian \& Owen, 1992)

38 The IED is an attentional set-shifting neuropsychological assessment from the

39 CANTAB battery. There are nine stages and the participant advances through the

40 stages by learning the "rule" at each stage. The number of stages completed was the

41 primary index used to measure executive function (Fagerlund, Pagsberg, \&

42 Hemmingsen, 2006; Luciana \& Nelson, 1998). 


\subsubsection{Cognitive Inhibition -Negative Priming Task}

The negative priming task is a computer generated modified version of the Stroop task based on a procedure outlined by Beech (Beech et al., 1989). Reaction times on a priming distractor (PD); neutral distractor (ND) and unrelated distractor (UD) are calculated and a negative priming effect calculated by subtracting PD-UD. The interference or Stroop effect is calculated by subtracting UD-ND.

\subsubsection{Spatial Working Memory: SWM (Sahakian \& Owen, 1992)}

10 Spatial working memory was assessed using the Cambridge Neuropsychological Test 11 Automated Battery. In this task a number of coloured boxes were displayed on the 12 screen with several of the boxes containing hidden tokens. The participant was 13 required to search through the boxes to find these hidden tokens. In each trial the participant, had to remember where the tokens had been located and search in alternate boxes. Strategies used by the participant to remember the location of the tokens were also recorded and each trial increased in difficulty. Between search errors occur when a participant returns to a box in which a token had already been found and strategy score based on the participant following a predetermined search sequence were the primary indicators used for analysis. A high strategy score indicated an inefficient strategy. All participants completed the battery however a small number of CC and NCC did not have their results recorded on IED/ SWM task due to faulty equipment.

\subsubsection{Divergent (creative) thinking task: Abbreviated Torrance Test of Adults (ATTA)} (Goff \& Torrance, 2002)). (Goff \& Torrance, 2002). This test comprises three tasks; the first calls for a verbal response to a hypothetical problem and the following two tasks require figural responses. Participants are asked to respond to the challenge using imagination and problem solving ability. Their responses are scored according to four normative referenced and fifteen criterion referenced indicators to obtain an overall creative indicator.

\subsubsection{Creative achievement: Creative Achievement Questionnaire (Carson, 2005).} To evaluate creative output, the Creative Achievement Questionnaire (CAQ; Carson, $2005 \# 4$ \} was administered to all participants. As young, developing professional creative artists were the focus of the creative participant group, an amended form of the CAQ questionnaire was administered to include relevant early achievement and achievement associated with contemporary outputs in online and digital creativity. 
1 In this pen and paper questionnaire, participants were asked to imagine sixteen

2 items and provide ratings of the vividness of their images using a five-point rating

3 scale where 5 indicated the least vivid image (no image at all) and 1 the most vivid

4 (perfectly clear and vivid as normal vision).

5

\subsection{Statistical Methods}

\section{Statistical analyses were conducted using SPSS 25. Descriptive analysis including} mean and SD were performed on all continuous variables and chi-square on categorical variables. ANOVA with Bonferroni correction was used to determine between group differences on all cognitive and creative variables. Effects of spatial working memory, vividness of mental imagery, IQ and IED were independently explored by a hierarchical regression. Creative achievement, as measured by the creative achievement questionnaire, was the outcome variable. Demographic covariables and then clinical predictors were entered into the model. For each step of the model, $\mathrm{R}$ square and $\mathrm{R}$ square change were reported. To further investigate the importance of spatial working memory relative to executive function/IQ and as a discriminatory variable, we conducted a stepwise discriminant functional analysis. Missing data was handled through listwise deletion.

\subsection{Results}

\subsubsection{Participants}

A total of one hundred and ten subjects participated in the study (PSD = 21; CVCC = 25 ; $C C=30 ; N C C=34$ ). No participants reported any visual impairment or colour blindness. The socio-demographic and clinical characteristics of the sample are shown in Table 1. More than half the participants were female (67\%) and the mean age was $23.9(S D=4.1)$. A total of $63.6 \%$ were students and had completed an average of $15.3(S D=2.09$ ) years of education. With respect to occupation, due to the small sample size the cells were recoded (part-time/unemployed vs other; $E P, C C x N C C$ ) (Fishers exact $p<.001$ ) and a significant difference was recorded. This was considered to be possibly indicative of the clinical and creative individual's unpredictable employment (Van den Eynde, Fisher, \& Sonn, 2016), however it was not deemed critical to further analysis. 


\section{Comparison between groups on cognitive and creative tests}

In Table 2, the ANOVA revealed a between group differences on creative thinking (ATTA), creative achievement (CAQ), vividness of mental imagery (VVIQ) and spatial working memory, between errors and strategy scores (Table 2). There were no significant differences between groups on IED, IQ and Negative Priming (NP) tasks. $I Q$, executive function, set shifting and cognitive inhibition did not discriminate EP and control groups. SWM between errors discriminated EP from all control groups however strategy was the only significant indicator of difference between EP and CVCC groups. These results indicate that compared to the early psychosis group, the clinically vulnerable creative group had significantly higher creative achievement and employed better spatial working memory strategy.

\section{Predicting creative achievement and group membership}

Results from the stepwise regression analysis indicated that while the cognitive factors of IQ, and executive function (IED) made some contribution to creative achievement, it was vividness of mental imagery $(B=.39, \rho<.01)$ that was the stronger predictor, contributing $16 \%$ of the variance.

Stepwise discriminant functional analysis was used to determine what variables; WASI IQ, executive function (IED total errors adjusted) and spatial working memory between errors and strategy best discriminated the four groups. Only the between errors spatial working memory variable significantly discriminated groups (Wilks' $\lambda=$ $0.85, \chi 2(1 \mathrm{~N}=$ ? $)=13.72, \mathrm{p}=0.003$; canonical discriminant coefficients: between search errors $=0.06$, constant $=-1.2$; functions at group centroids: $E P=0.74, C V C C=-$ $.34, \mathrm{CC}=-.21, \mathrm{NCC}=-0.10$ ). These results indicate that while spatial working memory is not a strong predictor of creative achievement, poor SWM does predict membership in the early psychosis group. 


\section{Discussion}

2 This research aimed to investigate specific cognitive differences between early

3 psychosis, clinically vulnerable creative, creative and non-creative populations. The

4 early psychosis, creative control and clinically vulnerable creative groups, compared to

5 non-creative controls, recorded significantly higher scores on creative potential (ATTA),

6 creative achievement (CAQ) and vividness of mental imagery (VVIQ). The clinically

7 vulnerable creative group performed better on creative cognition and achievement

8 than the other groups.

10 There were no significant differences on IQ, negative priming or executive function

11 (IED) across all four groups. This suggests that these global measures of cognitive

12 function do not discriminate between those in the early stages of psychotic illness,

13 those who may be more clinically vulnerable and those with no psychotic illness.

14 However it should be noted that as the psychosis population ages a significant decline

15 in executive function (abstraction), IQ and visual skills is reported (Fucetola et al., 2000)

16 and this is impacted by age at onset of the illness (Bora et al., 2009). Similarly, there

17 are differences in negative priming presentation between acute and those in remission

18 with inhibition being reduced in florid psychosis but restored in those under remission

19 (Minas \& Park, 2007). Within a younger psychosis population, at the early stage of

20 illness presentation and in remission, our results indicate that spatial working memory

21 was significantly impaired. This finding is in line with results from a similar study

22 (Goghari et al., 2014) discussed earlier, which found that between errors and strategy

23 on the CANTAB spatial working memory task differentiated UHR and FEP from HC.

24 Similarly, the early psychosis group differed from all three control groups on the

25 between errors score which measures short-term maintenance of visuospatial

26 memory; indicating some initial decline in visuospatial working memory. However, it

27 was only the clinically vulnerable creative group that recorded a significantly higher

28 strategy score when compared to the early psychosis group, indicating superior

29 executive function on visuospatial planning.

31 It has previously been suggested (Pirkola et al., 2005) that spatial working memory may

32 be a better neuro-cognitive marker for psychosis than verbal processing skills. In our

33 population, early psychosis participants recorded deficits in short term spatial working

34 memory. However, unlike the UHR group investigated in the Goghari study, the

35 creative clinically vulnerable group, alongside the creative and non- creative group

36 recorded similar results on between errors indicating stable short- term spatial

37 memory. Also, unlike the UHR group in the aforementioned study, the clinically

38 vulnerable creative group had more efficient strategy than the early psychosis group.

39 The creative and non-creative did not record any significant difference on strategy.

40 This is a measure of executive working memory that reflects planning and integration

41 of spatial information. Is it the superior ability to remember, plan and integrate spatial

42 information that assists the clinically vulnerable group to manage their heightened 
1 creative imagination and vivid mental imagery to achieve creatively in the presence of

2 positive and negative schizotypal symptoms and with sub clinical symptoms? The early

3 psychosis group in comparison has similar positive and negative schizotypal symptoms,

4 creative imagination and vivid imagery but has deficits in spatial memory and planning

5 and so was unable to achieve to the same extent creatively.

6

7 These results are considered in juxtaposition to the epidemiological study by McCabe

8 et al (2018), which concluded that artistic creativity was as significant risk factor for

9 psychotic disorders as other comparable risk factors. Our results support the view that

10 creative artists and early psychosis participants share personality and cognitive risk

11 factors. Our results point to spatial working memory as a possible cognitive "protective

12 factor" for the clinically vulnerable artistic student. While these results are tentative,

13 the role of spatial working memory as a possible cognitive factor that may prevent

14 transition to psychosis in vulnerable creative populations warrants further

15 investigation. One mechanism by which spatial working memory may act as a

16 protective factor for creative artists may be that as spatial working memory

17 deteriorates in early psychosis, vividness of imagery and creative imagination becomes

18 more dominant (Matthews et al., 2014) and so may lead to delusions. Carr (2010)

19 proposed the notion that delusion formation shares similarities to the creative process.

20 He suggests that delusions start with an aberrant proto-psychotic anomalous

21 experience in which the individual searches for meaning to explain the experience. This

22 search for meaning occurs under reduced pre-frontal functioning (such as when SWM

23 is impaired) where the delusion becomes the dominant belief. Carr likens this initial

24 proto-psychotic anomalous experience to the altered state and loosening of

25 associations that occurs in the initial stages of creativity (Csikszentmihalyi, 1996;

26 Eysenck, 1994) and supports other evidence that proposes a relationship between

27 creativity and risk for psychosis (Abraham, 2014). Following the initial loosening of

28 associations, he suggests that the second part of the creative process involves a

29 problem solving exercise to assign significance to this flight of ideas. It may be for the

30 creative individual, with intact spatial working memory, creative product occurs. For

31 the early psychosis individual with impaired spatial working memory (i.e., impaired

32 visual memory and problem solving ability), this may lead to inappropriate salience

33 assignment and delusions (Grillo, 2018).

35 Clearly more research into the role of spatial working memory with early psychosis

36 populations is needed. This study provides current evidence in support of the

37 aforementioned epidemiological findings (MacCabe et al., 2018) indicating creativity as

38 a vulnerability factor for psychosis. Our analysis identified an clinically vulnerable group

39 of creative artists in training who show similar schizotypal vulnerabilities to early

40 psychosis patients, without evidence of visuospatial cognitive decline. These findings

41 lend support to further longitudinal research into identifying and monitoring

42 vulnerable creative individuals in areas of schizotypy and spatial working memory. 


\section{Limitations}

3 These results should be interpreted in view of the limitations of the research. One of

4 the limiting factors for this study is the small sample size and cross-sectional

5 methodology; therefore without follow up data we cannot draw any conclusions or

6 implications on transition to psychosis. The clinically vulnearble creative control group

7 developed out of observing a pattern of symptomatology in a sub-set of creative

8 controls. Future research should seek to identify and provide longitudinal data on at

9 risk creative populations. Although the pilot study by Burkhardt et al did not advocate

10 screening for BP in creative populations, our research suggests that further longitudinal

11 studies are needed with this population to identify and determine risk over time. Due

12 to the small creative sample size we were not able to distinguish between different

13 domains of creativity for analysis. For example, are visual artists, rather than musicians

14 more clinically vulnerable and do visual artists have superior spatial working memory

15 and strategy compared to other creative artists? These are questions that warrant

16 further investigation.

18 Future Directions

19 This research supports the call for further research into visuo-cognitive impairments in

20 individuals at risk and individuals transitioning to psychosis. It also provides support for

21 innovative treatment protocols (Valmaggia, 2017) for early psychosis patients that use

22 visual mediums such as computer-based and virtual reality programs that may develop

23 and strengthen visuospatial working memory.

\section{Acknowledgements}

26 The authors would like to acknowledge Shannon Gostelow and Nicole O'Reilly who

27 were research assistants for the project.

28 The authors would also like to acknowledge the National Health and Medical Research

29 Council (NHMRC) Project grant (APP630471), and the Australian Research Council

30 (ARC) Future Fellowship (FT0991511) both grants held by Melissa J Green (UNSW).

31 Melissa J Green was responsible for this project as executed at UNSW and is affiliated

32 with UNSW, Neuroscience Research Australia (NeuRA), the Black Dog Institute, and the

33 Macquarie Centre for Excellence in Cognition and its Disorders.

35 Conflict of Interest

36 None 


\section{Data Availability statement}

The data that support the findings of this study are available on request from the corresponding author. The data are not publicly available due to privacy or ethical restrictions. 


\section{Bibliography}

Abraham, A. (2014). Is there an inverted-U relationship between creativity and psychopathology? Frontiers in Psychology, 5(750). doi:10.3389/fpsyg.2014.00750

Ando, V., Claridge, G., \& Clark, K. (2014). Psychotic traits in comedians. The British Journal of Psychiatry. doi:10.1192/bjp.bp.113.134569

Badcock, J. C., Michiel, P. T., \& Rock, D. (2005). Spatial working memory and planning ability: contrasts between schizophrenia and bipolar I disorder. Cortex, 41(6), 753-763.

Baddeley, A., D. (1996). Exploring the central executive. Quarterly Journal of Experimental Psychology, 18, 119-129.

Becker, G. (2014). A socio-historical overview of the creativity-pathology connection: from antiquity to contemparary times. In J. C. Kaufmann (Ed.), Creativity and Mental Illness (pp. 3-24). Cambridge: Cambridge University Press.

Beech, A., Powell, T., McWilliam, J., \& Claridge, G. (1989). Evidence of reduced cognitive inhibition in schizophrenia British Journal of Clinical Psychology, 28, 109-116.

Benson, T. L., \& Park, S. (2013). Exceptional visuospatial imagery in schizophrenia; implications for madness and creativity. Frontiers in Human Neuroscience, 7, 756.

Bora, E., \& Murray, R. M. (2013). Meta-analysis of Cognitive Deficits in Ultra-high Risk to Psychosis and First-Episode Psychosis: Do the Cognitive Deficits Progress Over, or After, the Onset of Psychosis? Schizophrenia Bulletin, 40(4), 744-755. doi:http://doi.org/10.1093/schbul/sbt085

Bora, E., Yucel, M., \& Pantelis, C. (2009). Cognitive functioning in schizophrenia, schizoaffective disorder and affective psychoses: meta-analytic study. The British Journal of Psychiatry, 195, 475-482.

0 Brewer, W. J., Wood, S. J., Phillips, L. J., Francey, S. M., Pantelis, C., Yung, A. R., . . McGorry, P. D. (2006).

Generalized and Specific Cognitive Performance in Clinical High-Risk Cohorts: A Review Highlighting Potential Vulnerability Markers for Psychosis. Schizophrenia Bulletin, 32(3), 538-555. doi:https://doi.org/10.1093/schbul/sbj077

Brod, J. (1997). Creativity and Schizotypy In G. Claridge (Ed.), Schizotypy: Implications for illness and health (pp. 274298). London: Oxford University Press.

Burkhardt, E., Pfennig, A., Breitling, G., Pfeiffer, S., Sauer, C., Bechdolf, A., . . Leopold, K. (2018). Creativity in persons at-risk for bipolar disorder-A pilot study. Early Intervention in Psychiatry, 1-8.

doi:https://doi.org/10.1111/eip.12748

Canivez, G. L., Konold, T. R., Collins, J. M., \& Wilson, G. (2009). The Wechler Abbreviated Scale of Intelligence and Wide Range Intelligence Test: Convergence and Structural Validity. School Psychology Quarterly, 24(4), 252265.

Carr, V. (2010). Beauty and belief: William James and the aesthetics of delusions in schizophrenia. Cognitive Neuropsychiatry, 15(1-3), 181-201. doi:http://doi.org/10.1080/13546800802332145

Carrión, R. E., Walder, D. J., Auther, A. M., McLaughlin, D., Zyla, H. O., Adelsheim, S., . . Cornblatt, B. A. (2018). From the psychosis prodrome to the first-episode of psychosis: No evidence of a cognitive decline. Journal of Psychiatric Research, 96, 231-238. doi:https://doi.org/10.1016/i.jpsychires.2017.10.014

Carson, S. H. (2011). Creativity and psychopathology: A Shared Vulnerability Model. Canadian Journal of Psychiatry, 56(3), 144-154.

Carson, S. H., Peterson, J. B., \& Higgins, D. M. (2003). Decreased latent inhibition is associated with increased creative achievement in high-functioning individuals. Journal of Personality and Social Psychology, 85(3), 499-506.

Castle DJ, Jablensky A, McGrath JJ, C. V., Morgan V, Waterreus A, Valuri G, . . A., F. (2006). The diagnostic interview for psychosis (DIP): development, reliability and applications. Psychological Medicine, 36 (1)(January), 69-80. 
Claridge, G., \& Blakey, S. (2009). Schizotypy and affective temperament: Relationships with divergent thinking and creativity styles. Personality and Individual Differences, 46(8), 820-826.

Csikszentmihalyi, M. (1996). Creativity Flow and the Psychology of Discovery and Invention. New York: Harper Collins.

Debbané, M., Badoud, D., Eliez, S., Conus, P., Schultze-Lutter, F., \& Flückiger, R. (2015). Developing Psychosis and Its Risk States Through the Lens of Schizotypy. Schizophrenia Bulletin, 41(suppl_2), S396-S407. doi:https://doi.org/10.1093/schbul/sbu176

Eysenck, H. J. (1994). Creativity and personality: Word association, origence, and psychoticism. Creativity Research Journal, 7(2), 209-216. doi:10.1080/10400419409534525

Fagerlund, B., Pagsberg, A. K., \& Hemmingsen, R. P. (2006). Cognitive deficits and levels of IQ in adolescent onset schizophrenia and other psychotic disorders. Schizophrenia Research, 85(1), 30-39. doi:https://doi.org/10.1016/j.schres.2006.03.004

Fucetola, R. P., Seidman, L. J., Kremen, W. S., Faraone, S. V., Goldstein, J. M., \& Tsuang, M. T. (2000). Age and neuropsychologic function in schizophrenia: a decline in executive abilities beyond that observed in healthy volunteers. Biological Psychiatry, 48, 137-146.

Goff, K., \& Torrance, E. P. (2002). Abbreviated Torrance Test for Adults (ATTA).

2 Goghari, V. M., Brett, C., Tabraham, P., John, L., Valmaggia, L., Broome, M., . . McGuire, P. (2014). Spatial Working Memory ability in individuals at Ultra High Risk for Psychosis. Journal of Psychiatric Research, 50(March), 100-105.

Grillo, L. (2018). A Possible Link between Anxiety and Schizophrenia and a Possible Role of Anhedonia.

Schizophrenia research and treatment, 2018, 5917475-5917475. doi:https://doi.org/10.1155/2018/5917475

Guilford, J. P. (1967). The nature of human intelligence. New York: MacGraw-Hill.

Keogh, R., \& Pearson, J. (2011). Mental Imagery and Visual Working Memory. PLOS ONE, 6(12), e29221. doi:https://doi.org/10.1371/journal.pone.0029221

Kyaga, S., Lichtenstein, P., Boman, M., Hultman, C., Langstrom, N., \& Landen, M. (2011). Creativity and mental disorder: family study of 300,000 people with severe mental disorder. British Journal of Psychiatry, 199, 373379.

Landgraf, S., \& Osterheider, M. (2013). "To see or not to see: that is the question." The "Protection-AgainstSchizophrenia" (PaSZ) model: evidence from congenital blindness and visuo-cognitive aberrations. Frontiers in Psychology, 4, 352-352. doi:https://doi.org/10.3389/fpsyg.2013.00352

Lecrubier, Y., Sheehan, D. V., Weiller, E., Amorim, P., Bonora, I., Harnett Sheehan, K., .. Dunbar, G. C. (1997). The Mini International Neuropsychiatric Interview (MINI). A short diagnostic structured interview: reliability and validity according to the CIDI. European Psychiatry, 12(5), 224-231. doi:doi: 10.1016/S0924-9338(97)83296-8 Luciana, M., \& Nelson, C. A. (1998). The functional emergence of prefrontal working memory systems in four-to eight-year-old children. Neuropsychologia, 36(3), 273-293.

MacCabe, J. H., Sariaslan, A., Almqvist, C., Lichtenstein, P., Larsson, H., \& Kyaga, S. (2018). Artistic creativity and risk for schizophrenia, bipolar disorder and unipolar depression: a Swedish population-based case-control study and sib-pair analysis. The British Journal of Psychiatry, 212(6), 370-376.

doi:https://doi.org/10.1192/bjp.2018.23

Marks, D. F. (1973). Visual imagery differences in the recall of pictures. British Journal Of Psychology (London, England: 1953), 64(1), 17-24.

Matthews, N. L., Collins, K. P., Thakkar, K. N., \& Park, S. (2014). Visuospatial imagery and working memory in schizophrenia. Cognitive Neuropsychiatry, 19(1), 17-35. doi:https://doi.org/10.1080/13546805.2013.779577 Michalica, K., \& Hunt, H. (2013). Creativity, Schizotypicality, and Mystical Experience: An Empirical Study. Creativity 56 Research Journal, 25(3), 266-279.

${ }_{58}^{5}$ Miller, G. F., \& Tal, I. R. (2007). Schizotypy versus openness and intelligence as predictors of creativity. Schizophrenia 
Minas, R. K., \& Park, S. (2007). Attentional window in schizophrenia and schizotypal personality: Insight from negative priming studies. Applied \& preventive psychology : journal of the American Association of Applied and Preventive Psychology, 12(3), 140-148. doi:10.1016/j.appsy.2007.09.003

Nelson, B., \& Rawlings, D. (2010). Relating Schizotypy and Personality to the Phenomenology of Creativity. Schizophrenia Bulletin, 36(2), 388-399. doi:10.1093/schbul/sbn098

Nettle, D. (2006). Schizotypy and mental health amongst poets, visual artists, and mathematicians. Journal of Research in Personality, 40(6), 876-890. doi:10.1016/j.jrp.2005.09.004

Oertel, V., Rotarska-Jagiela, A., van de Ven, V., Haenschel, C., Grube, M., Stangier, U., . . Linden, D. E. J. (2009). Mental imagery vividness as a trait marker across the schizophrenia spectrum. Psychiatry Research, 167(1), 1-11. doi:https://doi.org/10.1016/i.psychres.2007.12.008

Ottemiller, D. D., Elliott, C. S., \& Tania, G. (2014). Creativity, overinclusion and everyday tasks. Creativity Research Journal, 26(3), 289-296.

Parnas, J., Sandsten, K. E., Vestergaard, C. H., \& Nordgaard, J. (2019). Schizophrenia and Bipolar Illness in the Relatives of University Scientists: An Epidemiological Report on the Creativity-Psychopathology Relationship. Frontiers in Psychiatry, 10, 175.

42 Sass, L. A., \& Schuldberg, D. (2001). Introduction to the Special Issue: Creativity and the Schizophrenia Spectrum. $43 \quad$ Creativity Research Journal, 13(1), 1-4.

44 Siddi, S., Petretto, D. A., \& Preti, A. (2017). Neuropsychological correlates of schizotypy: a systematic review and meta-analysis of cross-sectional studies. Cognitive Neuropsychiatry, 22(3), 186-212. doi:10.1080/13546805.2017.1299702

Soeiro-de-Souza, M. G., Dias, V. V., Bio, D. S., Post, R. M., \& Moreno, R. A. (2011). Creativity and executive function across manic, mixed and depressive episodes in bipolar I disorder. Journal of Affective Disorders, 135(1-3), 292-297.

Srivastava, S., Childers, M. E., Baek, J. H., Strong, C. M., Hill, S. J., Warsett, K. S., . . Ketter, T. A. (2010). Toward interaction of affective and cognitive contributors to creativity in bipolar disorders: A controlled study. Journal of Affective Disorders, 125(1-3), 27-34. doi:10.1016/j.jad.2009.12.018

Thomson, P., \& Jaque, S. V. (2018). Childhood Adversity and the Creative Experience in Adult Professional Performing Artists. Frontiers in Psychology, 9, 111. doi:https://dx.doi.org/10.3389/fpsyg.2018.00111

Torrance, E. P. (1993). Understanding creativity: where to start? Psychological Inquiry, 4(3), 232-234. 9 Uhlhaas, P. J., \& Mishara, A. L. (2007). Perceptual anomalies in schizophrenia: integrating phenomenology and 
Valmaggia, L. (2017). The use of virtual reality in psychosis research and treatment. World psychiatry : official journal of the World Psychiatric Association (WPA), 16(3), 246-247. doi:https://doi.org/10.1002/wps.20443

Van den Eynde, j., Fisher, A., \& Sonn, C. (2016). Working in the Australian Entertainment Industry: Final Report. Retrieved from Victorian University, Melbourne, Australia

Wang, L., Xu, X., Wang, Q., Healey, G., Su, L., \& Pang, W. (2017). Are Individuals with Schizophrenia or Schizotypy More Creative? Evidence from Multiple Tests of Creative Potential. Creativity Research Journal, 29(2), 145156. doi:https://doi.org/10.1080/10400419.2017.1302777

Wechsler, D., \& Zhou, X. (2011). Manual for the Wechsler Abbreviated Scale of Intelligence. Bloomington, MN: Pearson Executive Office.

4 Yung, A. R., Yuen, H. P., McGorry, P. D., Phillips, L. J., Kelly, D., Dell'Olio, M., . . Buckby, J. (2005). Mapping the onset of psychosis: the Comprehensive Assessment of At-Risk Mental States. Australian and New Zealand Journal of Psychiatry, 39(11-12), 964-971. doi:https://doi.org/10.1111/j.1440-1614.2005.01714.x 
TABLE 1

\section{Sample description}

\begin{tabular}{|c|c|c|c|c|c|}
\hline & EP & CVCC & $\mathrm{CC}$ & NCC & Statistical Value for main effect \\
\hline$N$ & 21 & 25 & 30 & 34 & \\
\hline Age & $25.4 \pm 4.2$ & $23.8 \pm 4.8$ & $23.1 \pm 3.9$ & $23.7 \pm 3.7$ & $F_{2,111}=1.40, p=.246$ \\
\hline Female (\%) & 57.1 & 56.0 & 68.8 & 58.8 & $\chi^{2}(3 ; N=112)=1.06, p=.738$ \\
\hline Years of education & $14.95 \pm 2.78$ & $15.53 \pm 1.77$ & $14.56 \pm 2.39$ & $15.91 \pm 1.86$ & $F_{2,109}=1.07, p=.394$ \\
\hline Employment status & $\mathrm{N}(\%)$ & $\mathrm{N}(\%)$ & $\mathrm{N}(\%)$ & $\mathrm{N}(\%)$ & \\
\hline Student & $7(33.3)$ & $14(56.0)$ & $21(70.0)$ & $28(82.4)$ & \\
\hline Employee & $6(28.6)$ & $2(8.0)$ & $2(6.7)$ & $6(17.6)$ & \\
\hline Part time employment & $6(28.6)$ & $7(28.0)$ & $6(20.0)$ & $0(0)$ & \\
\hline Unemployed & $2(9.5)$ & $2(8.0)$ & $1(3.3)$ & $0(0)$ & \\
\hline PANSS - positive & $9.38 \pm 2.97$ & & & & \\
\hline PANSS - negative & $11.24 \pm 4.81$ & & & & \\
\hline PANSS - general & $21.00 \pm 4.16$ & & & & \\
\hline Age at onset & $16.58 \pm 2.91$ & & & & \\
\hline Age at first diagnosis & $19.10 \pm 3.53$ & & & & \\
\hline
\end{tabular}




\section{TABLE 2}

Means and standard deviations for all groups on creative thinking (ATTA), creative achievement (CAQ) and vivid imagery (VVIQ)

\begin{tabular}{|c|c|c|c|c|c|}
\hline & EP & CVCC & CC & NCC & $\begin{array}{l}\text { Statistical values for main } \\
\text { effects }\end{array}$ \\
\hline$N$ & 21 & 25 & 30 & 33 & \\
\hline WASI IQ & $107.9 \pm 15.2$ & $108.3 \pm 10.2$ & $110.2 \pm 10.2$ & $103.5 \pm 13.1$ & $F_{3,109}=1.8, p=.15 n s$ \\
\hline IED total errors adjusted & $22.67 \pm 20.94$ & $15.79 \pm 11.81$ & $26.83 \pm 22.13$ & $27.50 \pm 22.59$ & $F_{3,101}=1.83, p=.147 n s$ \\
\hline Negative Priming & $.0188 \pm .069$ & $-.0081 \pm .107$ & $-.0170 \pm .061$ & $-.0081 \pm .089$ & $F_{3,90}=.764, p=.517 n s$ \\
\hline Interference & $.0387 \pm .090$ & $.0297 \pm .075$ & $.041 \pm 060$ & $.0578 \pm .087$ & $F_{3,88}=1.26, p=.292 n s$ \\
\hline SWM Between errors & $29.6 \pm 21.6$ & $14.0 \pm 12.3$ & $14.8 \pm 11.9$ & $15.5 \pm 12.9$ & $F_{3,93}=5.42, p<.01^{\mathrm{a}}$ \\
\hline SWM Strategy & $32.9 \pm 4.6$ & $28.8 \pm 4.5$ & $29.7 \pm 4.7$ & $30.3 \pm 5.3$ & $F_{3,93}=2.92, p<.05^{b}$ \\
\hline ATTA & $76.5 \pm 12.5$ & $81.92 \pm 7.0$ & $77.4 \pm 9.9$ & $65.0 \pm 11.5$ & $F_{3,108}=14.5, p<.001^{\mathrm{c}}$ \\
\hline CAQ & $25.1 \pm 25.6$ & $55.8 \pm 32.1$ & $40.9 \pm 26.6$ & $2.6 \pm 4.1$ & $F_{3,108}=26.9, p<.001^{c, b}$ \\
\hline VVIQ & $62.8 \pm 12.0$ & $63.7 \pm 9.4$ & $63.3 \pm 9.3$ & $52.6 \pm 11.8$ & $F_{3,106}=7.6, p<.001^{c}$ \\
\hline
\end{tabular}

$\mathrm{a}=\mathrm{EP}>\mathrm{CC}, \mathrm{CVCC}, \mathrm{NCC}^{*}$

$\mathrm{b}=\mathrm{EP}>\mathrm{CVCC}^{*}$

$\mathrm{C}=\mathrm{EP}, \mathrm{CC}, \mathrm{CVCC} .>\mathrm{NCC} * *$

All analysis made with bonferroni correction 


\section{TABLE 3}

Stepwise regression with creative achievement as the dependant variable and $I Q$, executive function-set shifting, spatial working memory and vivid mental imagery as predictors.

\begin{tabular}{lllll}
\hline & B & SE B & B & P \\
\hline Step 1 & & & & \\
Constant & 18.51 & 19.67 & & .31 \\
\hline Age & .17 & .80 & .02 & .87 \\
Gender & 10.72 & 6.43 & .18 & .09 \\
Step 2 & & & & \\
\hline Constant & -39.80 & 31.58 & & .21 \\
Age & .21 & .78 & .03 & .80 \\
Gender & 10.87 & 6.26 & .18 & .08 \\
WASI IQ & .59 & .25 & .25 & .02 \\
IED & -.22 & .15 & -.16 & .14 \\
& & & & \\
Step 3 & & & & \\
Constant & -95.49 & 35.90 & & $<.01$ \\
Age & .06 & .72 & .01 & .93 \\
Gender & 9.89 & 5.82 & .16 & .09 \\
\hline WASI IQ & .57 & .23 & .24 & .02 \\
IED & -.25 & .14 & -.18 & .07 \\
SWM between errors & -.07 & .19 & -.04 & .73 \\
SWM strategies & .13 & .66 & .02 & .85 \\
Vivid mental imagery & .99 & .25 & .39 & $<.01$ \\
\hline
\end{tabular}

Note: $R^{2}=.03$ for step $1(p=.25) \Delta R^{2}=.08$ for step $2(p=.02) \Delta R^{2}=.16$ for step $3(p<.01)$ 
Appendix 1

TABLE 4

Schizotypy measured from Oxford Liverpool inventory of feelings and experiences (O-Life) and paranoid suspiciousness scale

\begin{tabular}{llllll}
\hline & EP & CVCC & CC & NCC & Statistical Value for main effect \\
\hline$N$ & 21 & 25 & 32 & 34 &
\end{tabular}

Positive (ImpNon) $\quad 9.57 \pm 4.87^{b} \quad 11.42 \pm 3.5^{b} \quad 7.84 \pm 3.71^{b} \quad \mathbf{5 . 0 9} \pm 3.4^{b} \quad\left(F_{3.98}=11.04, p<.001\right.$

Positive (UnExp) $\quad 12.38 \pm 7.59$ a $\quad 15.5 \pm 5.68$ a $\quad 11.50 \pm 6.38$ a $\quad 3.41 \pm 4.03^{a} \quad\left(F_{3.98}=16.28, p<.001\right.$

\section{Disorganised (CogDis) $\quad 14.81 \pm 5.14^{a} \quad 13.54 \pm 5.3^{a} \quad 11.06 \pm 4.78^{a} \quad 5.91 \pm 5.18^{a} \quad\left(F_{3.98}=13.07, p<.001\right.$}

\begin{tabular}{|c|c|c|c|c|}
\hline Negative (Paranoid) & $6.62 \pm 3.4^{b}$ & $6.36 \pm 2.44^{b}$ & $4.75 \pm 2.65^{b}$ & $\begin{array}{ll} & \left(\left(\mathrm{FF}_{3.99}=7.36, p<.001\right.\right. \\
\mathbf{3 . 1 8} \pm \mathbf{2 . 6 8} \text { b }^{\prime} & \left(\mathrm{F}_{3.99}=7.36, p<.001\right.\end{array}$ \\
\hline
\end{tabular}

$\mathrm{a}=\mathrm{EP}, \mathrm{CVCC}, \mathrm{CC}>\mathrm{NCC} * * \mathrm{~b}=\mathrm{EP}, \mathrm{CVCC}>\mathrm{NCC} * * *$ Schizotypy measured from O-Life and Paranoid suspiciousness scale 